\title{
The Application of Blockchain in Supply Chain Industry
}

\author{
Leo Willyanto Santoso ${ }^{1}$, Yulia ${ }^{2}$ \\ ${ }^{1,2}$ Infomatics Department, Petra Christian University, Surabaya, Indonesia \\ ${ }^{1}$ leow@petra.ac.id
}

Article History: Received: 10 November 2020; Revised: 12 January 2021; Accepted: 27January 2021;

Published online: 05April 2021

\begin{abstract}
This research focuses on analyzing the state of the art of the blockchain technology and in publicizing the potential applications and implementations that the blockchain can deliver to the supply chain. For this, it will be explained in a simple way technology and its operation in the supply chain and case studies of real companies that are currently betting on this technological revolution, capable of improving the efficiency of any process logistic.

Keywords:Blockchain, Supply Chain, Efficiency, Logistic
\end{abstract}

\section{Introduction}

Blockchain is the technology that supported the well-known cryptocurrency Bitcoin, invented by Satoshi Nakamoto in 2008. For years the chain of blocks was unknown to society, but after the cryptocurrency boom, many scientists and developers were interested in this technology. Although it wasn't until the year 2015, when nine finance companies (Barclays, BBVA, UBS, JPMorgan, etc.) partnered to develop technology-based financial services test platforms Blockchain [1]. From then on, almost daily announcements of new startups that implemented the blockchain appeared in fintech services. It took a little longer for the community specialized in supply chain management, realized the impact that Blockchain could have on the industry.

The main premise was to create transparency, all members of the network have access to the same data, which provides a single truth [2]. It collects in their writings reflections of different authors that deal with the potential of Blockchain technology [3]. Thus, they consider that the transparency of the supply chain is one of the most relevant and most difficult areas to achieve [4]. Not surprisingly, some logistics experts consider that the blockchain offers "enormous potential" [5].

Blockchain would be a "very necessary platform for economic renewal" was predicted [6] and that, it is capable of transforming the supply chain and modify the way in which we produce, market, buy and we consume our products" [7].

However, as is often the case with emerging technology, the hype around Blockchain network seems mainly promoted by technology developers, consultants and journalists. Logistics operators, especially small and medium-sized companies, claim to have little knowledge about Blockchain technology [8]. This can be explained through the theory of diffusion innovation [9], but also because of the lack of convincing real use cases that clearly show the benefit of applying the blockchain above other existing Information Technology services and solutions.

For these reasons, this research proposes the following objectives. In first place, clarify the concept, characteristics, foundations and operation of Blockchain in a simple and clear way for any reader to be able to understand this complex and novel technology. The second objective is to investigate the various applications that the blockchain can have in all the processes that make up the supply chain and what are the benefits it is able to bring to the management of the herself. And as a last objective, illustrate and encourage medium and small companies to participate in this technological revolution, through real cases of large organizations who are currently implementing the Blockchain system in their operations, achieving efficiency improvements in your processes and considerable cost savings.

\section{Blockchain}

Blockchain or chain of blocks is a distributed database designed in order to avoid manipulation and modification once a data has been posted using a reliable timestamp and linking to a previous block. In others words, Blockchain is an immutable digital book that records all transactions that are perform on the network safely thanks to encryption. 
What makes the Blockchain so secure is that all the information with all the operations in the databases are fragmented and stored in different locations (distributed ledger). That is, all the data is divided into pieces of equal size that are housed in the computers connected to the network, which act as servers. So to steal information or manipulate it, the entire system should be hacked, which is impossible. Also, the fact that the data is fragmented and distributed makes the information public and verifiable by anyone, although always anonymously.

In 2008, Blockchain was born from the hand of the cryptocurrency Bitcoin, a digital currency decentralized distribution, that is, it does not depend on any entity or body that regulate it like a bank or a government could. Cryptocurrencies are totally dependent on Blockchain for its operation, since it is the chain of blocks that allows the registration of all the operations that are made with them. Bitcoin is the most cryptocurrency recognized. All its popularity came when the value of one unit of this coin went from being worth just three US dollars in March 2013 to reaching a value, in less than five years. This fact led to the rise of cryptocurrencies and over the years they have gone developing hundreds of them, such as Ether, Litecoin, and ADA.

All these transactions are protected by a cryptographic method. Every operation which is validated, is sealed in data blocks that are grouped together. These groups or chains of blocks, which are also protected with the aforementioned cryptography, generates a record immutable and "unreachable" that is saved, as we have mentioned before, in fragments on different computers anywhere in the world. This security system so sophisticated is what generates a hopeful feeling about Blockchain.

Currently, a real technological revolution is taking place that will mean a change radical in the exchange of value, this innovation is Blockchain.

Society has always tried to minimize the uncertainty associated with any process that requires decisionmaking. When society based all its economic activity in hunting and gathering, all mercantile exchange was limited to closest environment. But as civilizations grew and commerce began its apogee, the first organisms were established to participate in the activity economic: governments, banks, companies, societies,... These institutions were the precursor tools of intervention in the economy, with the aim of reducing the growing uncertainty generated by the lack of control that the growth of trade entailed worldwide.

Until one fateful day in August 2007, the Lehman Brothers bank fell. This success it led to the beginning of a global economic crisis. Some of the items are the causes of the crisis were errors in economic regulation, speculation, corruption and the inappropriate practices of banking entities. It was this motive that prompted Satoshi Nakamoto to develop, a year later, Bitcoin, and consequently, Blockchain. Gathering previous failed studies on virtual money, managed to develop an electronic system decentralized that did not have to depend on third parties [10].

Today, at the height of e-commerce, supported to a large extent by commerce international, single intervention by economic institutions and government may not be enough. For the first time in history, institutions traditional must be pushed aside to make room for a new technological tool.

Blockchain, capable of controlling the uncertainty that the current technological wave is causing in which we are immersed.

\section{SWOT Analysis of Blockchain}

\section{A. Strengths}

Among the strengths of Blockchain, the most unstable quality is the decrease in costs. Today, the role of the intermediary is necessary to make any transaction more reliable, since it is the figure that intervenes between the two parties, ensuring and guaranteeing that the contract is fulfilled and that everyone is satisfied. But this leads to an increase in cost monetary and temporary of any operation. Blockchain allows eliminating the figure of intermediary and third parties, thanks to the solidity and immediacy. Such is the security that protects this technology that, in its ten years of life, the Blockchain network has never been hacked [11].

Other advantages that the blockchain boasts is security and transparency. As it is a distributed ledger, the information about the transactions is shared to all the nodes connected to the network and, after its consensus and validation, it passes to be part of a block in the chain in an immutable way, so the data will be always available for future consultation. This information will be one hundred percent reliable, since before being added to the 
blockchain, it must be verified by half plus one of the nodes connected to the network. The basis of this technology is consensus:

If all users have the same information, it must be true. Also, Being immutable, the data can never be altered, so the information is more rigorous, robust and transparent.

Another positive feature of Blockchain is consistency. Having connected thousands of users as nodes from anywhere in the world, the system will be in continuous operation, so the network will never fail or suffer drops. Also, the number of nodes and the proper functioning of the blockchain is directly proportional, that is, the more users there are, the more robust the Blockchain will be.

The last notable strength is immediacy. As seen above, not being the figure of third parties is necessary, there are no intermediaries that slow down the process, whether banks, companies or any other body. If two parties want to make a transaction, when the set conditions are met, the conditions are automatically executed, without the assessment and interpretation of a third party may be necessary.

\section{B. Weaknesses}

Blockchain has a series of weaknesses inherent to its own operation that must be resolved or improved so that this technology acquires the merit that belongs to it.

One of the biggest drawbacks of the blockchain is linked to one of its main benefits. Blockchain is so secure because all the nodes that are connected to the network receive a copy of each transaction, but this is a great loss energy and time, since the same process must be repeated in all nodes to reach to consensus. All the necessary procedure for the network to function properly is much heavier than if it were carried out in a single centralized node.

The Blockchain database is constantly increasing, every time a block is validated and is added to the chain. This leads to a necessary increase in computational requirements necessary for correct operation, since the nodes must each time support a greater weight of blockchains. What causes an unconscious centralization of the process, because, over time, higher technical requirements are needed generating entry barriers for any user who wants to participate in the Blockchain.

As mentioned above, another of the main problems of Blockchain is the speed. Currently, a block has a maximum storage capacity of $1.30 \mathrm{MB}$ (megabyte), as can be seen in Graph 1, which shows the evolution of the average capacity since April 2018. The average weight of a transaction is $0.5 \mathrm{~kb}$ (kilobyte), so that the same block can store a maximum of 2,660 transactions, approximately. Blockchain adds a new block to a chain every ten minutes, so that this implies that, currently, four transactions per second are averaged in Blockchain. This is ridiculous compared to, for example, 1,667 transactions per second that VISA averages [12].

Another notable disadvantage is the energy consumption involved in data mining. How was developed earlier, miners need computer equipment with high power computer workers who work twenty-four hours, seven days a week, consuming huge amounts of energy. All the benefits of virtual technology can offer the environment thanks, among other improvements, to the reduction of the use of paper, is called into question due to the high energy consumption involved in the operation of the Blockchain network.

The last weakness that we are going to review is the lack of maturity. The European Union establishes some parameters of technological maturity or Technology Readiness Level (TRL)[13]. Currently, Blockchain is at the fifth level, which implies that this technology "is ready to integrate in systems and the algorithms can run on processors with similar characteristics to those of an operating environment" [13], but it implies that the network is still in development and cannot yet be applied in real situations.

\section{Opportunities}

As has been exposed, Blockchain suffers from serious threats that they can make jeopardizing the stability and future of this technology. Despite this, there are a number of future opportunities that must be exploited to the maximum to take full advantage of them and overcome all the adversities that hinder the development of the blockchain. 
Blockchain is raising a lot of buzz around it, as it has the potential enough to bring about significant progress in social justice, education system and health, distribution of purchasing power or human rights. Blockchain technology proposes a more efficient model of society and the economy, since, by improving transparency, traceability and citizen participation, it is possible to reduce corruption, disloyal and increase the satisfaction of society in general [14].

Another of the great strengths that the blockchain can achieve is a development unprecedented in transparency and traceability, due to intrinsic properties of Blockchain, which does not allow the manipulation of information and makes it relatively public. Blockchain technology allows the end consumer to know each component, piece, additive or element that has been added to the product since it has been cultivated, raised or manufactured until the customer has purchased it.

Currently, the usual systems to carry out financial transactions are through credit or debit card, PayPal or bank transfers. These platforms carry ample commissions, in addition to adding a period of time for the operation to be completed.

Another of the great opportunities of the Blockchain network eradicates this system. There are hundreds of payment platforms that stand out for their security, their immediacy and their nullity of commissions by not depending on intermediaries that handle users' money. Furthermore, these platforms work with cryptocurrencies. The advantage of these digital currencies that is decentralized, so they do not depend on any government, institution or bank for their circulation. This allows an independence never seen in the own and unique money management.

The last opportunity that is going to be analyzed, and which is surely the most interesting and the more scalable, are the possible applications of various kinds of Blockchain. Its robustness and reliability make it a system that can be implemented in many areas, both in the sector public and private, creating new business models. The blockchain has applications in the health system, creating a record of patient history on the network that would improve coordination between hospitals, and even to learn about new diseases or develop drugs. Also, it can be implemented in government agencies such as home registration, thus avoiding possible fraud in the sale of properties. The education system would be another sector where technology Blockchain could perfectly merge. A clear example of this is Tutellus, which is a decentralized online training platform, whose objective is to provide educational resources to students who lack the means to train and offer rewards to educators who join the platform [15].

But the implementation of the blockchain that is of greater interest and that is the reason for carrying out this research are the Blockchain applications in the supply chain. The integration of chained block technology in the logistics operations is capable of rewriting the current definition of distribution.

\section{Threat}

Despite the evident progress that would imply the implementation of Blockchain technology in society and the economy, is currently haunted by several serious threats that try to stop the development of the blockchain and that must be confronted.

The biggest drawback that Blockchain must overcome is the mentality of the players, both governments as organizations. For Blockchain to work properly, all parties involved should renew their systems for registering and treating information. That is, governments, banks, companies and society in general would have to collaborate by sharing information on the blockchain. This consensus will depend on large measure of the perception that is had of the risk-benefit ratio that Blockchain can contribute.

The theory of diffusion of innovation develops how an idea, product, service is adopted or technology in a society over time. Adoption occurs at different rates according to the perceptions of each individual or organization, therefore, five categories: innovators, early adopters, early majority, late majority and laggards.

This theory is easily applicable to blockchain technology. Countries like Japan, Switzerland or The Netherlands have regulated Blockchain technology and accepted cryptocurrencies as legal tender. Other countries, such as the United Kingdom and South Korea, are investing in research centers that study how to implement Blockchain in their systems to get the most out of it and become the future reference for this technology, even grant economic facilities to emerging companies in the sector. On the contrary, China and the 
United States are very reluctant to adopt this system, imposing requirements regulations in order to slow down their development, until a common regulation is studied.

From here another obstacle can be glimpsed, the lack of regulation. The biggest challenge will be getting to harmony between states. Legislation should be imposed that defends the weakest and that does not allow the use of Blockchain for fraudulent activities.

Another threat to highlight is speculation. Currently, the majority of users of Blockchain participate in the network because of the large speculative market it represents. As mentioned above, initially, the chain of blocks was born to support the cryptocurrencies. The best known as Bitcoin or Ether, are granted to data miners as a reward for validating blocks that later become part of the chain.

There is a limited number of cryptocurrencies so the offer is scarce, while the demand is high [12]. This causes a huge fluctuation in the value of the same, in a few days, the price can rise or fall radically. This makes Blockchain very attractive to investors, although, in turn, it is detrimental to the network since, if the value grows a lot, transactions will become more expensive and will grant less reward to miners who will look for another system with better incentives, and with them users, as the network will slow down.

\section{Application of Blockchain Technology in Supply Chain}

According to the theoretical study analyzed above, with blockchain technology you can develop distributed information platforms where traceability can be verified of a good from its manufacture to the end of its passage through the supply chain, avoiding centralization, thus making it easier to trust and cooperate among all members participating in the process. With the collaboration of all of them, the chain of blocks, along with the Internet of Thing implementation and together with smart contracts, will revolutionize the way information is recorded and managed, thus achieving benefits for the supply chain, such as automation of transactions such as payments and collections, guarantee in the fulfillment of contracts, security and control of logistics operations, or obedience to laws and regulations.

The theory is overwhelming, such a system is capable of achieving an extraordinary improvement in the performance and efficiency. Due to its inherent characteristics of immutability and security, Blockchain has the ability to optimize the flow of information, stock and capital, obtaining a reduction in time, costs and risks.

Commercial distribution stands out for the heterogeneity and fragmentation of the sector. Due to this, the study of Blockchain applications to the supply chain will be sectioned according to the utility of the applications depending on four fundamental sectors: pharmaceutical, automotive, food and public. The importance of efficient management of the supply chain in these four sectors has been the reason for their specific choice for further analysis. Furthermore, it is in these industries that it is currently possible find a greater number of organizations that research and invest in development and implementation of Blockchain technology.

\section{E. Application of Blockchain in Pharmaceutical Industry}

According to the World Health Organization, it is estimated that each year they are sold globally Counterfeit pharmaceuticals worth up to 200 billion US dollars and that $50 \%$ of these drugs are purchased over the Internet [16]. Counterfeiting, in general, occurs when a manufacturer or distributor recalls a part of the drugs produced and resells them, or introduces between the original batches counterfeit drugs. The problem is that the technological solutions that are taking, they are not able to dispel these illicit acts. Pharmaceutical companies, increasingly, are more subdued by governments. For example, in 2013, the Congress of the States The United States enacted the Drug Quality and Safety Act, in order to improve the patient safety. This law requires that, by 2024, all pharmaceutical companies guarantee the full traceability of prescription drugs throughout the supply chain. Current technology is not enough for compliance with these laws, in favour of drug safety, be real. To do this, it seems necessary to give a twist, being Blockchain a great candidate to solve these puzzles.

Product tracking refers to tracking items at the unit level, from start to end of its passage through the supply chain. Thanks to the Blockchain application, all Participants in the exchange can access the origin and location, authenticate the elements that comprise it and verify compliance with the requirements and agreements. This possible, thanks to the distribution of information in real time, an inherent characteristic of the Blockchain platform. For example, the blockchain will allow tracking of medicines throughout their life cycle, from manufacture to consumption by of patients, facilitating the identification of counterfeit drugs, supporting the management of the withdrawal of the same; thus increasing pharmacological safety [17]. 
Thus, achieving complete traceability and at any time $[18,19]$. Any defect, such as temperature variation in the refrigeration of any stored medicine, will be captured by an IoT device and this information will be stored and tracked on the chain of blocks through smart contracts. Automatically, actions will be executed according to the rules and conditions that have been configured in the smart contracts and notify all participating members of the supply chain of the event.

Smart contracts are coded to perform a specific reaction for each action that may happen, this will give companies the ability to automatically respond to any event, failure or problem that may occur. For example, if medications are not met certain previously defined conditions, they could be withdrawn before their market launch, thus achieving not only compliance with the contract, but also preventing the sale of potentially dangerous products for patients, benefiting society in general.

Another application of Blockchain technology is the digital signature. This will allow a follow-up Accurate and responsible of medicines during their passage through the supply chain, decentralizing the entire process, by eliminating the need for certification by a only authorized source.

Blockchain platforms may be used to store information and enforce regulatory standards to the letter during the distribution of drugs. From a regulatory perspective, the solutions offered by the blockchain will provide a reliable source of information for authorities regulations that will be able to retrieve a complete history of the life cycle of a medicine. In addition, pharmaceutical companies will be able to boast of compliance with the standards of quality without question.

\section{F. Application of Blockchain in Automotive Industry}

The automotive industry has a complex structure, due to the large number of stages and intermediaries involved in the car life cycle process. In addition to be a sector that needs continuous technological innovation. This is why some of the big car brands such as Volkswagen, Mercedes or Ford, among others, are studying how the technology offered by the blockchain could be implemented in various purposes: driverless cars, electric vehicles, chain of supply or financial services.

The application of Blockchain in the supply chain of the automotive sector would have benefits tangible for all actors involved in the chain. The final consumer could access to a distributed, secure and immutable database, where you can check the entire cycle of life of the vehicle you wish to purchase. Users will have the opportunity to verify in any time in a reliable and transparent way, the state of the vehicle, the origin of raw materials, quality of components and accessories, repairs and modifications that it has undergone or any event or incident during its passage through the supply chain. This could clear up doubts and facilitate customer purchase decision process.

The pertinent government authorities will be able to access real and truthful information about the cars that are currently circulating on national roads or that are going to be put for sale to the public. Thanks to these databases, made possible by the Blockchain network, You will be able to check any information about the vehicles: accumulated mileage, date of registration, reviews carried out, polluting emissions, etc. With all the information that the authorities can collect, sustainable development strategies can be proposed To reduce pollutant emissions, plans that try to reduce accidents of traffic and increase road safety or measures to eradicate theft and illicit trafficking of automobiles.

The insurance companies will handle more reliable information on the way of driving each user, being able to offer fairer insurance modalities, such as "pay as you drive".

Other big beneficiaries would be producers and distributors, since they will be able to guarantee the quality of the products and components used in the manufacture of vehicles and will achieve a more efficient and precise distribution thanks to automation and coordination that allows the implementation of the chain of blocks in the supply chain, reducing operating costs and thus achieving greater added value to the customers.

In practice, one of the possible uses of Blockchain in the supply chain by automobile companies, would be the constitution of an open and shared market for the purchase and sale of materials and components taking advantage of the functionalities of smart contracts. These could store the information about supply and demand market, facilitate supply decision making and generate requests for buyers [17]. At that time, the providers that use the platform will receive a request and may submit an offer. The manufacturer can then select automatically to a supplier (for example, the first one that meets the requirements set) or select a dealer manually. The 
advantage offered by the contract intelligent, in addition to automation, is the possibility of establishing conditions. By For example, the deterioration of the merchandise or the delay in the supply could be punished with a fine that will not depend on subjective assessments, due to automatic execution of the clauses previously set.

\section{G. Application of Blockchain in Food Industry}

In Indonesia, the food sector continues to grow year after year. In 2018, according to the Federation of Indonesia Food and Beverage Industries, food and beverage production reached a value of 116,890 million dollars. Thus, its contribution to the economy national represents $3 \%$ of GDP and $16 \%$ of the total of the Indonesia industrial sector, generating at the end of 2018 , a number of 426,300 jobs.

It should be noted, in this context, that due to events not so distant as the disease of mad cows and bird flu, society began to become aware of the importance of has food security. This is why, like the pharmaceutical industry, the food sector is under great pressure from government institutions to comply with food safety protocols that guarantee the correct conditions hygienic-sanitary and food disposal. In the American continent, the Law of Modernization of Food Safety, introduced in 2011 by the Administration Food and Drug Administration, aimed to take steps to prevent food contamination instead of trying to combat it [20].

To respond correctly to the influx of regulatory standards on food, by changing consumer demands and increased competition, companies belonging to the food industry must test, exploit and exploit the technologies disruptive, such as Blockchain, which are capable of administering and managing the activities of the supply chain in a more efficient way, maximizing profits, and guaranteeing, in turn, total transparency.

The traceability and transparency of the supply chain are of vital importance to ensure proper food health safety, so it is necessary to know all the factors that can affect the quality and condition of the food that the final consumer. For this reason, institutions such as the Spanish Association of Commercial Codification have developed a platform that allows manufacturers, wholesalers and retailers to share easily information on products throughout their supply chain journey in a safe way.

The first level where to implement Blockchain would be in production and collection (ranchers, farmers, fishermen, etc.), responsible for obtaining the necessary raw material for the elaboration of the product. The platform would store detailed information on the product obtained by the producer, responding to all the Possible questions: what, when, where, how, with what or how much. Thus, it would be possible to have absolute control of all the factors that have influenced their development during development early stages of the food life cycle. For example, you would get data from chemicals used in agricultural harvesting and in what quantity, or how they have been fed and raised the animals and birds from which dairy products and eggs are obtained for human consumption. Thus, avoiding the consumption of foods that contain allergens and pathogens that carry disease, greatly improving safety food.

The second step in the chain, where to implement Blockchain, would be the industrial sector, since the raw material is received and transformed into the final product suitable for consumption. Here, the Food is handled, transformed and manipulated, during this process the product it can be contaminated, deteriorated or altered. For this reason, traceability acquires vitally important: all companies must be able to have comprehensive control of the processes, following the imposed parameters, with a constant and updated flow of information in real time. The implementation of the blockchain on a platform information would make it easier to control processes such as: dates of entry and exit of batches, origin of raw material, cooking or storage temperatures, detailed data on sweeteners, preservatives, additives and chemicals in general, packaging conditions and storage, transport of goods or contamination caused by the process productive.

The distribution would be the last stage of the supply chain where the chain of blocks would have utility. It is the last step before the food reaches the final consumers, then a distributor would have the opportunity to check the traceability of the product.

\section{Conclusion}

It is essential that organizations conduct a comprehensive internal assessment prior to implement Blockchain in your operations to ensure risk mitigation associated with technology. Issues such as the level of market adoption or possible regulatory constraints, raise a number of concerns for most technologies emerging. These risks, such as a dubious competitive advantage or integrity of security, may make some companies think twice 
before moving forward towards the implementation of Blockchain in their operations. However, concerns more frequent, detailed below, can be mitigated with proper planning:

- Visibility of the source: the concern on the part of companies is that the competition can see the details of the supply chain source.

- Supply chain security: The concern in this case is that the use of a distributed ledger digital ledger may carry increased risk of attack cybernetic.

- Volume of transactions: organizations are suspicious that any competitor can determine the quantity and value of the merchandise being transported. Without However, the content of a trail on the blockchain can be encrypt, making it impossible to know this data.

A thorough evaluation of the above considerations will likely alleviate the risks associated with the implementation of Blockchain in the supply chain. Above From all other considerations, creating a strategy based on the chain of blocks, will support entities in the management and development of solutions that solve existing challenges and creating operational efficiency gains.

The ability of Blockchain technology in supply chain management is an issue increasingly popular, with proofs of concept developed across industries, such as it has been demonstrated in the previous chapters. The first companies to participate in this revolution, they will have the opportunity to work as a team with regulators and technology developers, gaining the opportunity to shape how technology Blockchain will work in your industries, setting the rules for this new game.

The public generally only relates the blockchain to Bitcoin or cryptocurrencies and this means a step backwards for technology, since not a few governments and entities claim, several successfully, criminalize the use of cryptocurrencies, an attitude largely caused by high speculation and their decentralization. Adoption by society It will depend on the risk-benefit ratio transmitted by the Blockchain technology and, for this, It must be able to offer an independent image and away from cryptocurrencies. How currently, the data they are the oil of the 21st century, and whoever dominates the Blockchain, will have a huge advantage competitive over others.

\section{References}

1. S. Underwood, "Blockchain beyond Bitcoin," Communications of the ACM 59(11):15-17 2016.

a. Tapscott and D. Tapscott, "How blockchain is changing finance," Harvard Business Review, 1 March 2017.

2. N. Hackius and M. Petersen. "Blockchain in logistics and supply chain: trick or treat?" Hamburg International Conference of Logistics 2017.

3. S. Abeyratne and R. Monfared, "Blockchain Ready Manufacturing Supply Chain Using Distributed Ledger," International Journal of Research in Engineering and Technology,05(09),pp.1-10.

4. K. O'Marah, Blockchain For Supply Chain: Enormous Potential Down The Road. URL: https://www.forbes.com/sites/kevinomarah/2017/03/09/blockchain-for-supplychain-enormouspotential-down-the-road/.

5. M. J. Casey and P. Wong, "Global Supply Chains Are About to Get Better, Thanks to Blockchain," Harvard Business Review, 13 March 2017.

6. B. Dickson, Blockchain Has the Potential to Revolutionize the Supply Chain. URL: https: //techcrunch.com/2016/11/24/blockchain-has-the-potential-to-revolutionize-thesupply-chain/.

7. W. Kersten, M. Seiter, B. von See, N. Hackius, and T. Mauer, "Trends and Strategies in Logistics and Supply Chain Management - Digital Transformation Opportunities,” Project: Trends and Strategies in SCM and Logistics - Opportunities of the digital transformation.

8. E. M. Rogers, Diffusion of Innovations, 5th Edition, Free Press, 2003.

9. P. Champagne, "The Books of Satoshi: The collected Writings of Bitcoin Creator Satoshi Nakamoto," E53 Publishing LLC, 372 p. 2014.

a. Batra, R. Olson, S. Pathak, N. Santhanam, and H. Soundararajan, "Blockchain 2.0: What's in store for the two ends-semiconductors (suppliers) and industrials (consumers)?," McKinsey Advanced Electronics, 18 January 2019.

10. T. M. Fernández-Caramés and P. Fraga-Lamas, "A Review on the Use of Blockchain for the Internet of Things," in IEEE Access, vol. 6, pp. 32979-33001, 2018

11. D. Vidal-Tomás and A. Ibañez, "Semi-strong efficiency of Bitcoin," Finance Res. Lett., 27 (2018), pp. 259-265.

12. M. Dobrovnik, D. M. Herold, E. Fürst, and S. Kummer, "Blockchain for and in Logistics: What to Adopt and Where to Start," Logistics 2018, 2, 18 
13. S. Sharma and R. S. Batth, "Blockchain Technology for Higher Education Sytem: A Mirror Review," 2020 International Conference on Intelligent Engineering and Management (ICIEM), London, United Kingdom, 2020, pp. 348-353.

14. M. Montecchi, K. Plangger, and M. Etter, "It's real, trust me! Establishing supply chain provenance using blockchain,” Business Horizons, Vol. 62, Issue 3, May-June 2019, 283-293.

15. L. Kehoe, N. O'Connell, D. Andrzejewski, K. Gindner, and D. Dalal, "When two chains combine: Supply chain meets blockchain," Deloitte.

16. S. DeCovny, "Experts Discuss Tackling Pharma Supply Chain Issues With Blockchain," URL: http://www.nasdaq.com/article/experts-discuss-tackling-pharma-supplychain-issues-with-blockchaincm808938.

17. T. K. Mackey and G. Nayyar, "A review of existing and emerging digital technologies to combat the global trade in fake medicines," Expert Opinion on Drug Safety 16(11), March 2017

18. FDA (2011). Food Safety Modernization Act (FSMA). U.S. Food and Drug Administration (FDA). https://www.fda.gov/food/guidance-regulation-food-and-dietarysupplements/food-safetymodernization-act-fsma

19. B.Nammari, "Blockchain in Government - Major Research Paper (MRP)," Project: The Strategy of Implementing Blockchain to Build the Smart Government/City, May 2017.

20. Turashvili, M. (2018). Using Blockchain in Georgia. National Agency of Public Registry, Georgia. https://www.elra.eu/wp-content/uploads/2018/06/Mariam-Turashvili-NAPRBlockchain-Presentation3.0.pdf 